\title{
Cancer Immunotherapy and Personalized Medicine: Emerging Technologies and Biomarker-Based Approaches
}

Laura Maciejko ${ }^{1 \#}$, Munisha Smalley ${ }^{1 \#}$ and Aaron Goldman ${ }^{1,2,3 *}$

${ }^{1}$ Integrative Immuno-Oncology Center, Mitra Biotech Woburn, MA 01801, USA

${ }^{2}$ Department of Medicine, Harvard Medical School, Boston, MA 02115, USA

${ }^{3}$ Division of Engineering in Medicine, Department of Medicine, Brigham and Women's Hospital, Boston, MA 02115, USA

"Equal contribution

\begin{abstract}
Purpose of review: The vision and strategy for the 21 st century treatment of cancer calls for a personalized approach in which therapy selection is designed for each individual patient. While genomics has led the field of personalized cancer medicine over the past several decades by connecting patient-specific DNA mutations with kinase-targeted drugs, the recent discovery that tumors evade immune surveillance has created unique challenges to personalize cancer immunotherapy. In this mini-review we will discuss how personalized medicine has evolved recently to accommodate the emerging era of cancer immunotherapy. Moreover, we will discuss novel platform technologies that have been engineered to address some of the persisting limitations.

Recent finding: Beginning with early evidence in personalized medicine, we discuss how biomarker-driven approaches to predict clinical success have evolved to account for the heterogeneous tumor ecosystem. In the emerging field of cancer immunotherapy, this challenge requires the use of a novel set of tools, distinct from the classic approach of next-generation genomic sequencing-based strategies. We will introduce new techniques that seek to tailor immunotherapy by re-programming patient-autologous T-cells, and new technologies that are emerging to predict clinical efficacy by mapping infiltration of lymphocytes, and harnessing fully humanized platforms that reconstruct and interrogate immune checkpoint blockade, ex-vivo.

Summary: While cancer immunotherapy is now leading to durable outcomes in difficult-to-treat cancers, success is highly variable. Developing novel approaches to study cancer immunotherapy, personalize treatment to each patient, and achieve greater outcomes is penultimate to developing sustainable cures in the future. Numerous techniques are now emerging to help guide treatment decisions, which go beyond simple biomarker-driven strategies, and are now we are seeking to interrogate the entirety of the dynamic tumor ecosystem.
\end{abstract}

Keywords: Tumor ecosystem; Cancer immunotherapy; Precision diagnostics; Biomarker

\section{Introduction}

According to the National Cancer Institute (NCI) there were $1,685,210$ new cancer diagnoses in 2016 [1]. With such a high number of patients, effective treatment to halt the progression and/or cure cancer is of primary concern. Traditionally, cancer patients are prescribed a predetermined first line treatment depending on the cancer indication and grade. As our understanding for the complexity and uniqueness of each individual tumor improves, the suitability of the this 'one-sizefits-all' approach to cancer therapy is called into question. Precision medicine, the proposed future for the treatment of disease, is based on a tailored approach for selecting therapy at the individual patient level [2]. Given the high patient-to-patient variability, cancer is a prime candidate for the use of precision medicine. Indeed, it is important that the first line therapy is effective since relapse can reduce 5 -year survival rates by more than $50 \%$ in aggressive cancers [3]. It is now clear that the future of personalized cancer treatment will involve a complete biochemical characterization of the tumor using multi-dimensional analyses for a range of biological endpoints, which will lead to a calculated decision on the appropriate treatment. Thus, resulting in significantly improved Overall Response (ORR) and Overall Survival (OS) rates.

The recent discovery that tumors co-opt immune check-points as a mechanism of escaping immune surveillance has led to a renaissance in immunotherapy, and revolutionized cancer treatment options [4,5]. This suggests that inherent patient-specific biology is likely driving responses [6,7] which is thought to be largely dependent on the global immune system of each individual patient, and not necessarily dependent only on the tumor biology [8]. Therefore, new technologies are needed that can help predict which patients will benefit from immunotherapy. While cancer immunotherapy has eliminated terminal disease in some patients, clinical success is highly variable [9]. Indeed, the emerging paradigm of cancer immunotherapy is bringing with it added complexity to the challenge of personalized cancer medicine, and an answer for it that has yet to be fully elucidated [6,7,10-13]. Effective implementation of personalized cancer medicine is limited by the lack of well-established preclinical models and methods that integrate global tumor heterogeneity, with high fidelity, in terms of the role of cancer and stromal cells, tumor microenvironment, 3-D architecture, and immune contexture [14]. Indeed, current 'gold standard' in-vitro and ex-vivo preclinical approaches that interrogate drugs using cell lines and spheroids [15-17] or ex-vivo organotypic tumor models are all limited by their inability to capture the full biological approximation of the native tumor, resulting in poor mapping to clinical outcomes [17-20].

Here, we will highlight some of the pervading strategies in personalized medicine, and further elucidate how research is moving beyond the "one size fits all" approach to treatment selection.

*Corresponding author: Aaron Goldman, Division of Engineering in Medicine Department of Medicine, Brigham and Women's Hospital, Boston, MA 02115, USA Tel +1 617-432-1000; E-mail: goldman1@mit.edu; agoldman@mitrabiotech.com

Received April 28, 2017; Accepted June 26, 2017; Published June 28, 2017

Citation: Maciejko L, Smalley M, Goldman A (2017) Cancer Immunotherapy and Personalized Medicine: Emerging Technologies and Biomarker-Based Approaches. J Mol Biomark Diagn 8: 350. doi: 10.4172/2155-9929.1000350

Copyright: (c) 2017 Maciejko L, et al. This is an open-access article distributed under the terms of the Creative Commons Attribution License, which permits unrestricted use, distribution, and reproduction in any medium, provided the original author and source are credited. 


\section{Classic Biomarker-Based Approaches for Precision Diagnostics}

Discovery of molecular cancer biomarkers (i.e., cancer 'addictive' oncogenes) has paved the way for the first generation of personalized therapy. Indeed, genomic screening approaches have been commonly employed to identify tumor-specific, overexpressed proteins or genetic mutations that may confer mechanisms of therapeutic resistance in cancer cells [21]. Targeting these protein biomarkers therapeutically can lead to better clinical outcomes. For example, antibody and smallmolecule inhibitors of specific proteins, such as the Human Epidermal Growth Factor Receptor 2 (HER2) has led to successful implementation of diagnostic tools like HercepTest $[22,23]$. The discovery of HER2 overexpression in other indications has led to the approval, in 2010, of Trastuzumab for gastric or gastroesophageal junction adenocarcinoma [24]. Interestingly, the FDA-approval of existing therapies for new indications is common, with the repurposing of thalidomide to treat multiple myeloma being a notable example $[25,26]$.

Similarly, the overexpression of EGFR in various types of cancer was discovered as early as 1997 [27]. This led to the development of specific EGFR tyrosine kinase inhibitors (EGFR-TKIs) such as gefitinib or afatinib. However, acquired resistance to these therapies occurs in approximately $60 \%$ of patients treated with first line EGFR-TKIs [28]. It has subsequently been found that a specific mutation of EGFR is responsible for this acquired resistance, T790M. This mutation has also been observed in other types of EGFR-TKI-resistant lung cancers. The recently FDA approved small molecule inhibitor, osimertinib (Tagrisso), is an EGRF-TKI and is most effective in patients with this acquired EGFR mutation, and is used following failure of first line EGFR-TKI therapies [29]. More recently, midostaurin (Rydapt), a small molecule inhibitor of VEGF, has been approved for use in acute myeloid leukemia (AML) patients with mutated FMS-like tyrosine kinase inhibitor 3 (FLT3) [30,31]. With only $10 \%$ of leukemia patients and $25 \%$ of AML patients presenting with mutations in FLT3, biomarkers are becoming increasingly useful to connect patients to the correct therapy [32]. As one example, the companion diagnostic LeukoStrat CDx FLT3 mutation assay, was FDA-approved alongside midostaurin in April 2017, and the Phase III trial showed an improved OS rate of $23 \%$ [33].

While the biomarker-driven approach for treatment decision making in the clinic usually yields better than standard of care results, with an improvement on ORR and OS rates, there are currently only 16 drugs out of more than 200 FDA-approved agents that require, or benefit from a companion diagnostic test [34]. Moreover, the emerging generation of new anticancer therapies, which seek to invigorate the body's own immune system, suggests that new approaches for personalized medicine must evolve.

\section{Targeting the Immune Compartment}

\section{Cytokine stimulation}

William Coley, commonly referred to as one of the pioneers of cancer immunotherapy, recognized the link between a patient's complete remission from sarcoma with a Streptococcus pyogenes infection in 1890 [35]. This led to him treating cancer patients with bacteria post-surgery, resulting in an immune response that would keep any tumor resurgence at bay. Although this approach was largely unsuccessful due to the adverse effects of the infection, it demonstrated that activating the immune response could be of therapeutic benefit in cancer. It was not until 1992 that the first immunotherapy was approved by the FDA; high dose interleukin-2 (HD IL-2), for renal cell carcinoma
(RCC) [36]. From 1986 to 2006, the NCI treated 259 metastatic RCC patients with HD IL-2. The objective response rate was just $20 \%$, with 23 patients achieving a complete response and 30 patients achieving a partial response [36]. A similar response rate was observed with HD IL-2 treatment in melanoma, which was FDA-approved in 1998 [37]. This non-specific immunotherapeutic approach is one of very few therapies that can result in complete remission, albeit in a small cohort of patients.

\section{Immune checkpoint inhibitors}

Immune checkpoints are negative regulators of $\mathrm{T}$ cell immunity, usually inhibiting the stimulation of $\mathrm{T}$ cells. The primary function of immune checkpoint inhibition is to rescue $\mathrm{T}$ cells from exhaustion or deplete $\mathrm{T}$ regulatory cells $\left(\mathrm{T}_{\text {reg }}\right)$. Indeed, the best characterized immune cells in cancer biology are $\mathrm{CD}^{+}$helper T-cells, which exacerbate tumor proliferation, and cytotoxic $\mathrm{CD}^{+} \mathrm{T}$-cells, which have been shown to prevent tumor growth [38]. One of the many receptors involved in immune checkpoints, cytotoxic T-lymphocyte-associated protein 4 (CTLA-4), discovered in 1987, modulates the extent of $\mathrm{T}$ cell activation by competitively binding to B7 proteins, which are required for stimulation of T cells. However, it was not until 1996 that targeting CTLA- 4 was shown to have anti-cancer effects in mice [39]. This seminal study led to the development of the first immune checkpoint inhibitor, ipilimumab (Yervoy), an anti-CTLA-4 monoclonal antibody, which was approved in 2011 for metastatic melanoma. The success of ipilimumab led to the development of even more efficacious immune checkpoint inhibitors such as pembrolizumab (Keytruda) and nivolumab (Opdivo), PD-1 inhibitors, and atezolizumab (Tecentriq) a PD-L1 inhibitor. Merck's pembrolizumab has been particularly successful, being FDA-approved for a range of indications, including metastatic melanoma, metastatic non-small cell lung cancer (NSCLC) and refractory Hodgkin's Lymphoma. A recent report of a phase III trial showed 10.3 months progression free survival for untreated advanced NSCLC treated with pembrolizumab, compared with 6 months for platinum-based chemotherapy [40]. Moreover, in May 2017, the FDA approved pembrolizumab for the treatment of solid tumors found to be microsatellite instability-high or mismatch repair deficient. This is the first time the FDA has approved a therapy based on a biomarker rather than the location of the cancer, exemplifying the progression of personalized medicine. With 734 on-going clinical trials for immunotherapies combined with other therapies, the clinical potential of immune checkpoint inhibitors is yet to be fully realized.

\section{Adoptive cell transfer: Personalized T-cell therapy}

Chimeric antigen receptor (CAR)- $\mathrm{T}$ cells are an example of adoptive cell transfer (ACT). CAR T cells taken from a patient (or other human) are engineered to express cancer-specific antigens ex vivo and are administered back into the patient. CAR-T cell therapy has shown efficacy in the treatment of many B cell malignancies, most notably against B cell acute lymphoblastic leukemia (B-ALL). In a study with 53 children and young adults with CD19+ ALL, 50 out of 53 patients went into complete remission following treatment with CAR-T cells [41]. Despite these incredible results in liquid cancers, treatment using CAR-T cells is more difficult in solid tumors, perhaps due to physical and biochemical differences. In a phase I clinical study, patients with EGFR-positive relapsed/refractory NSCLC were treated with EGFR-targeted CAR-T cells. Of 11 evaluable patients, only two patients showed a partial response and five had stable disease for two to eight months [42]. There have been proposed theories to further improve CAR-T cell therapy such as enhancing the selectivity of the 


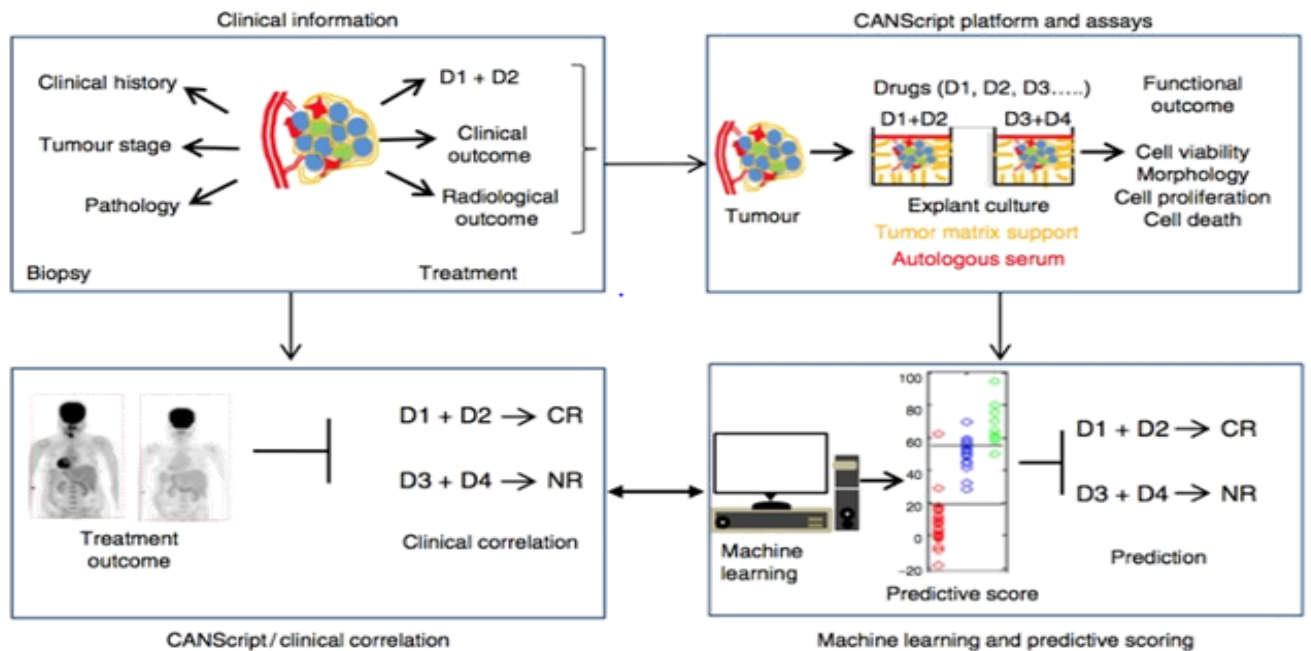

Figure 1: CANscript ${ }^{\circledR}$ platform technology. Four critical modules were integrated in generating and validating the CANscript platform. The first module involved collecting tumor core or surgical biopsy with tumor staging and/or pathology information besides clinical history. In the second module, tumor biopsy was rapidly processed into thin explants. The explants were cultured with tumor- and grade-matched TMPs and autologous serum (AS) and incubated with selected drug regimens. While multiple drug regimens can be used, the one used by the oncologist for the patient was always included in the tumor explant culture. The in vitro functional outcome of treatment in terms of cell viability, pathological and morphological analysis, cell proliferation, and cell death was quantified. In module three, these quantitative scores from the explants were aggregated using a machine learning algorithm to assign a final score, which helped rank the outcomes as complete response (CR), partial response $(\mathrm{PR})$, or no response (NR). In the final module, these predictions were tested against clinical outcomes. D1, D2, D3, and D4 indicate different drug regimens (image courtesy of Mitra RxDx).

CAR and using this therapy in conjunction with immune checkpoint inhibitors [43]. Whilst CAR-T cell therapy is very promising, there are many noted negative side effects, including the potentially fatal cytokine release syndrome, which also have to be addressed if this therapy is to be adopted for wider use.

Another example of ACT in the clinic is the expansion of patient tumor infiltrating lymphocytes (TIL) with IL-2, ex vivo, with subsequent administration directly into the tumor. This approach has resulted in promising OS rates in the clinic. It has been used to treat B cell malignancies and melanoma with great success in a small subset of patients. Moreover, in a recent study, 3 out of 9 of metastatic cervical cancer patients treated with human papillomavirus targeted-TIL responded; 2 of which experienced complete regression lasting up to 22 months [44].

\section{Biomarkers for Personalized Cancer Immunotherapy}

While tumor associated biomarkers have been employed to personalize kinase-targeted agents for each patient (as described in more detail above), these same approaches have been less robust for personalized cancer immunotherapy. Given the complexity of the tumor microenvironment, and the heterogeneous cellular landscape, there is a clear need to improve upon our classic biomarker-based approaches to incorporate the entirety of the tumor ecosystem, and tumor-immune contexture $[7,45]$.

In the context of the immune system, the ability for T-cells to mount a response to invasive growth of tumors is associated with improved outcome to therapy [46]. Several studies have now established that spatial distribution and infiltration of lymphocytes into tumors can predict progression-free or overall survival [47]. Indeed, lymphocytes of two distinct subclasses $\left(\mathrm{CD}^{+} / \mathrm{CD}^{+}, \mathrm{CD}^{+} / \mathrm{CD} 45 \mathrm{RO}^{+}\right.$, or $\mathrm{CD}^{+} / \mathrm{CD} 4 \mathrm{RO}^{+}$) will confer distinct infiltration patterns that map to distinct predictive outcomes [47]. Halio Dx, a French cancer diagnostics company, has created the first standardized immune-based assay Immunoscore ${ }^{\star}$ Colon for quantifying spatial heterogeneity of infiltrating immune cells, Immunoscore ${ }^{\star}$ Colon [48]. Specific for colon cancer, the test requires a tumor core and invasive margin, which are studied to determine the distribution and density of both $\mathrm{CD}^{+} \mathrm{T}$ lymphocytes and $\mathrm{CD} 8^{+}$cytotoxic T cells. An algorithm is used to generate their Immunoscore (I4 indicating the most cytotoxic and memory immune infiltrate and I0 indicating the lowest) [48]. Groups of patients were defined based on microsatellite status and Immunoscore and then analyzed for survival. Regardless of microsatellite status, patients with an Immunoscore of I3 and I4 had prolonged disease-specific survival (DSS) and OS. In addition, patients with a score between I0-I2 showed higher risk of relapse, shorter DSS and OS [49]. This approach, investigating the number and density of infiltrating lymphocytes, has clearly shown to be beneficial and better than existing measures of response, it is important to note that the immune marker expression profile of immune cells can be a valuable predictor of response. For instance, in clinical trials using ipilimumab, melanoma patients with higher expression of genes involved in immune function, including CD8A, CD27, CD38, CD3, CD40, GZMB, PRF1, CCL4, were more likely to have positive response to treatment [50]. Despite these advances, evidence for dynamic mechanisms of resistance have led to new speculation about how to personalize cancer immunotherapy $[4,10]$.

Mitra Mitra RxDx Inc., a personalized cancer medicine company based in Boston, Massachusetts, created an ex vivo tumor model, which harnesses fresh tumor biopsies and surgical excisions to study the effect of drugs in a fully humanized, autologous context, termed CANscript ${ }^{\circ}$ [51]. CANscript ${ }^{\circ}$ preserves the cellular and microenvironment heterogeneity by maintaining the tumor tissue in the presence of autologous plasma and peripheral blood nucleated cells (PBNC) in tissue culture wells coated with cancer type and grade-matched tumor matrix proteins (TMP). These elements enable accurate re-capitulation of the 3D architecture of the tumor and the tumor microenvironment, including the immune compartment. This ex-vivo platform has been used to evaluate the functional effects of conventional cytotoxic and kinase targeted cancer therapies. Using a series of endpoints following drug 
Citation: Maciejko L, Smalley M, Goldman A (2017) Cancer Immunotherapy and Personalized Medicine: Emerging Technologies and BiomarkerBased Approaches. J Mol Biomark Diagn 8: 350. doi: 10.4172/2155-9929.1000350

Page 4 of 5

exposure. This was used to train a machine learning algorithm which generates an 'M-score' for each drug tested, which is predictive for drug response (Figure 1).

Excitingly, this platform demonstrates the first use for dynamic cellular heterogeneity, and real-time analysis of the tumor-immune contexture under pressure of immune checkpoint inhibitors. For example, Mitra recently demonstrated the flexibility of the platform to the assay to capture changes to the T-cell immune repertoire, and help predict the clinical efficacy of multiple immuno-oncology agents, such as immune checkpoint inhibitors [52,53]. Indeed, by mapping the role of T-regulatory $\left(\mathrm{T}_{\mathrm{reg}}\right)$ cells in the presence or absence of multiple PD-1 checkpoint blockade, their research team elucidated that antitumor outcomes can be unique within a single patient sample. They discovered that depletion of $\mathrm{T}_{\text {reg }}$, but not increase of IFN-g expressing cytotoxic T-cells correlates to antitumor response. These findings are the first to support patient-specific response to a single immunotherapy among multiple drugs that target the same immune checkpoint. Such revelations could help shape the future of treatment selection in immunotherapy at the individual patient level.

\section{Concluding Remarks}

Cancer treatment decision-making is the final frontier in our challenge to cure cancer. While biomarkers, oncogenes and mutations have been classic maps for predicting therapy outcome, clinical success has reached a plateau. Even in the new age of cancer immunotherapy, target-specific molecular characteristics of a tumor are only present in a small proportion of patients. Immunotherapies can also cause immune-related adverse events, especially with CTLA-4, which can be serious and affect a large proportion of treated patients, with one trial observing it in one third of patients [54]. This makes prior patient testing even more vital to ensure these powerful next generation treatments are prescribed to the correct population.

The current problem is that the mechanism by which a drug target is selected is dictated by our knowledge of the role of growth factor receptors or key immune checkpoint proteins, and not patient-specific biology. There are a multitude of overexpressed, mutated and/or dysfunctional proteins in a tumor. Targeting one may not yield the best response rates for patients. There needs to be a way of deciding the relative efficacy of multiple treatment options for patients, in order to be sure of giving them the best possible chance of surviving and overcoming their cancer. We are in the midst of an exciting time for the development of truly personalized medicine, with the emergence of technologies that have the capacity to predict clinical outcomes. A more unbiased approach to drug selection has been sought with the invention of high throughput sequencing, searching the whole genome for mutations, but there is still, a disconnect between the discovery of mutations and deciding which drug is administered. The discovery of new platform technologies, which go beyond molecular biomarkers by seeking to interrogate the dynamic and heterogeneous microenvironment which go beyond characterizing individual biomarkers may lead to improved personalized approaches for cancer immunotherapy. Not only do these types of platforms ensure that the patient gets the best treatment for their specific type of cancer, but it also paves the way for the discovery of drug efficacy in tumors that might not otherwise have been tested, leading to new drug discovery or repurposing of immunotherapy with conventional drugs.

\section{Funding}

The funding agencies that are supportive for the research:

1. NIH RO1 (1R01CA135242)

\section{Breast Cancer Alliance Young Investigator Award}

3. American Cancer Society Postdoctoral Fellowship (122854-PF-12-226-01-CDD).

\section{References}

1. Siegel RL, Miller KD, Jemal A (2016) Cancer statistics. CA Cancer J Clin 66: 7-30.

2. Collins FS, Varmus $H(2015)$ A new initiative on precision medicine. $\mathrm{N}$ Engl J Med 372: 793-795.

3. Carey LA (2011) Directed therapy of subtypes of triple-negative breast cancer Oncologist 1: 71-78.

4. Mellman I, Hubbard-Lucey VM, Tontonoz MJ, Kalos MD, Chen DS, et al. (2016) De-risking immunotherapy: Report of a consensus workshop of the cancer immunotherapy consortium of the Cancer Research Institute. Cancer Immuno Res 4: 279-288.

5. Page DB, Postow MA, Callahan MK, Allison JP, Wolchok JD (2014) Immune modulation in cancer with antibodies. Annu Rev Med 65: 185-202.

6. Wayteck L, Breckpot K, Demeester J, De Smedt SC, Raemdonck K (2014) A personalized view on cancer immunotherapy. Cancer Lett 352: 113-125

7. Kakimi K, Karasaki T, Matsushita H, Sugie T (2017) Advances in personalized cancer immunotherapy. Breast Cancer 24: 16-24.

8. Spitzer MH, Carmi Y, Reticker-Flynn NE, Kwek SS, Madhireddy D, et al. (2017) Systemic immunity is required for effective cancer immunotherapy. Cell 168 487-502 e15.

9. Ferris RL, Blumenschein G Jr, Fayette J, Guigay J, Colevas AD, et al. (2016) Nivolumab for recurrent squamous-cell carcinoma of the head and neck. $N$ Engl J Med 375: 1856-1867.

10. Deng X, Nakamura $Y$ (2017) Cancer precision medicine: From cance screening to drug selection and personalized immunotherapy. Trends Pharmacol Sci 38: 15-24.

11. Gupta SK, Jaitly T, Schmitz U, Schuler G, Wolkenhauer O, et al. (2016) Personalized cancer immunotherapy using systems medicine approaches. Brief Bioinform 17: 453-467.

12. Kidd BA, Readhead BP, Eden C, Parekh S, Dudley JT (2015) Integrative network modeling approaches to personalized cancer medicine. Per Med 12: 245-257.

13. Ophir E, Bobisse S, Coukos G, Harari A, Kandalaft LE (2016) Personalized approaches to active immunotherapy in cancer. Biochim Biophys Acta 1865: $72-82$

14. Tannock IF, Hickman JA (2016) Limits to personalized cancer medicine. N Eng J Med 375: 1289-1294

15. Garnett MJ, Edelman EJ, Heidorn SJ, Greenman CD, Dastur A, et al. (2012) Systematic identification of genomic markers of drug sensitivity in cancer cells. Nature 483: $570-575$

16. Samson DJ, Seidenfeld J, Ziegler K, Aronson N (2004) Chemotherapy sensitivity and resistance assays: A systematic review. J Clin Oncol 22: 3618-3630.

17. Sharma SV, Haber DA, Settleman J (2010) Cell line-based platforms to evaluate the therapeutic efficacy of candidate anticancer agents. Nat Rev Cancer 10: 241-253.

18. Jung PS, Kim DY, Kim MB, Lee SW, Kim JH, et al. (2013) Progressionfree survival is accurately predicted in patients treated with chemotherapy for epithelial ovarian cancer by the histoculture drug response assay in a prospective correlative clinical trial at a single institution. Anticancer Res 33 : 1029-1034.

19. Ridky TW, Chow JM, Wong DJ, Khavari PA (2010) Invasive three-dimensional organotypic neoplasia from multiple normal human epithelia. Nat Med 16 1450-1455.

20. Vaira V, Fedele G, Pyne S, Fasoli E, Zadra G, et al. (2010) Preclinical model of organotypic culture for pharmacodynamic profiling of human tumors. Proc Natl Acad Sci USA 107: 8352-8356.

21. Sethi S, Ali S, Philip PA, Sarkar FH (2013) Clinical advances in molecular biomarkers for cancer diagnosis and therapy. Int J Mol Sci 14: 14771-14784.

22. Barrett C, Magee H, O'Toole D, Daly S, Jefferset M (2007) Amplification of the HER2 gene in breast cancers testing 2+ weak positive by HercepTest immunohistochemistry: False-positive or false-negative immunohistochemistry? J Clin Pathol 60: 690-693. 
Citation: Maciejko L, Smalley M, Goldman A (2017) Cancer Immunotherapy and Personalized Medicine: Emerging Technologies and BiomarkerBased Approaches. J Mol Biomark Diagn 8: 350. doi: 10.4172/2155-9929.1000350

23. Selvarajan S, Osamuraet RY (2004) The HercepTest and routine C-erbB2 immunohistochemistry in breast cancer: Any difference? Ann Acad Med Singapore 33: $473-476$

24. Gunturu KS, Woo Y, Beaubier N, Remotti HE, Saif MW (2013) Gastric cance and trastuzumab: First biologic therapy in gastric cancer. Ther Adv Med Oncol 5: 143-151.

25. Barlogie B (2001) Extended survival in advanced and refractory multiple myeloma after single-agent thalidomide: Identification of prognostic factors in a phase 2 study of 169 patients. Blood 98: 492-494.

26. Scannell JW, Blanckley A, Boldon H, Warrington B (2012) Diagnosing the decline in pharmaceutical R\&D efficiency. Nat Rev Drug Discov 11: 191-200.

27. Rusch V, Klimstra D, Venkatraman E, Pisters PW, Langenfeld J (1997) Overexpression of the epidermal growth factor receptor and its ligand transforming growth factor alpha is frequent in resectable non-small cell lung cancer but does not predict tumor progression. Clin Cancer Res 3: 515-522.

28. Yu HA, Arcila ME, Rekhtman N, Sima CS, Zakowski MF, et al. (2013) Analysis of tumor specimens at the time of acquired resistance to EGFR-TKI therapy in 155 patients with EGFR-mutant lung cancers. Clin Cancer Res 19: 2240-2247.

29. Sgambato A, Casaluce F, Maione P, Rossi A, Rossi E, et al. (2012) The role of EGFR tyrosine kinase inhibitors in the first-line treatment of advanced nonsmall cell lung cancer patients harboring EGFR mutation. Curr Med Chem 19 3337-3352.

30. Levis M (2017) Midostaurin approved for FLT3-mutated AML. Blood 129: 3403 3406.

31. Walker AR, Wang H, Walsh K, Bhatnagar B, Vasu S, et al. (2016) Midostaurin bortezomib and MEC in relapsed/refractory acute myeloid leukemia. Leuk Lymphoma 57: 2100-2108.

32. Deschler B, Lubbert M (2006) Acute myeloid leukemia: Epidemiology and etiology. Cancer 107: 2099-2107.

33. Gupta A, Viswanatha DS, Patnaik MM (2017) FLT3 mutation testing in acute myeloid leukemia. JAMA Oncol 3: 991

34. Myers MB (2016) Targeted therapies with companion diagnostics in the management of breast cancer: Current perspectives. Pharmgenomics Pers Med 9: 7-16

35. Coley WB (1893) The treatment of malignant tumours by repeated inoculations of erysipelas with a report of ten original cases. Am J Med Sci 105: 487.

36. Klapper JA, Downey SG, Smith FO, Yang JC, Hughes MS, et al. (2008) High-dose interleukin-2 for the treatment of metastatic renal cell carcinoma: A retrospective analysis of response and survival in patients treated in the surgery branch at the National Cancer Institute between 1986 and 2006 Cancer 113: 293-301.

37. Bhatia S, Tykodi SS, Thompson JA (2009) Treatment of metastatic melanoma: An overview. Oncology SSSS23: 488-496.

38. Alderton GK (2012) Tumour immunology: Suppressing tumorigenic inflammation. Nat Rev Cancer 12: 228.

39. Leach DR, Krummel MF, Allison JP (1996) Enhancement of antitumor immunity by CTLA-4 blockade. Science 271: 1734-1736.
40. Reck M, Rodríguez-Abreu D, Robinson AG, Hui R, Csőszi T, et al. (2016) Pembrolizumab versus chemotherapy for PD-L1-Positive non-small-cell lung cancer. N Engl J Med 375: 1823-1833.

41. Onea AS, Jazirehi AR (2016) CD19 chimeric antigen receptor (CD19 CAR) redirected adoptive T-cell immunotherapy for the treatment of relapsed or refractory B-cell Non-Hodgkin's Lymphomas. Am J Cancer Res 6: 403-424.

42. Feng K, Guo Y, Dai H, Wang Y, Li X, et al. (2016) Chimeric antigen receptormodified $T$ cells for the immunotherapy of patients with EGFR-expressing advanced relapsed/refractory non-small cell lung cancer. Sci China Life Sci 59: $468-479$.

43. Jin C, Yu D, Essand M (2016) Prospects to improve chimeric antigen receptor T-cell therapy for solid tumors. Immunotherapy 8: 1355-1361.

44. Stevanović S, Draper LM, Langhan MM, Campbell TE, Kwong ML, et al. (2015) Complete regression of metastatic cervical cancer after treatment with human papillomavirus-targeted tumor-infiltrating T cells. J Clin Oncol 33: 1543-1550.

45. Yuan J, Hegde PS, Clynes R, Foukas PG, Harari A, et al. (2016) Novel technologies and emerging biomarkers for personalized cancer immunotherapy $\mathrm{J}$ Immunother Cancer 4: 3 .

46. Fridman WH, Pagès F Sautès-Fridman C, Galon J (2012) The immune contexture in human tumours: Impact on clinical outcome. Nat Rev Cancer 12: $298-306$

47. Galon J, Costes A, Sanchez-Cabo F, Kirilovsky A, Mlecnik B, et al. (2006) Type density, and location of immune cells within human colorectal tumors predict clinical outcome. Science 313: 1960-1964.

48. Hermitte F (2016) Biomarkers immune monitoring technology primer: Immunoscore(R) Colon. J Immunother Cancer 4: 57

49. Mlecnik B, Bindea G, Angell HK, Maby P, Angelova M, et al. (2016) Integrative analyses of colorectal cancer show immunoscore is a stronger predictor of patient survival than microsatellite instability. Immunity 44: 698-711.

50. Ji RR, Chasalow SD, Wang L, Hamid O, Schmidt H, et al. (2012) An immuneactive tumor microenvironment favors clinical response to ipilimumab. Cancer Immunol Immunother 61: 1019-1031.

51. Majumder B, Baraneedharan U, Thiyagarajan S, Radhakrishnan P, Narasimhan $\mathrm{H}$, et al. (2015) Predicting clinical response to anticancer drugs using an ex vivo platform that captures tumour heterogeneity. Nat Commun 6: 6169.

52. Radhakrishnan P, Sekar VK, Brijwani N, Chevour P, Balakrishnan B, et al (2017) A patient derived ex vivo platform CANscript predicts distinct therapeutic outcomes to multiple PD-1 checkpoint inhibitors in single tumor biopsies. Cancer Res 77: 3681.

53. Majumder B, Ulaganathan B, Thayakumar A, Thiyagarajan S, Brijwani N, et al. (2015) Identification of responders for Anti-CTLA4 in refractory colorectal cancers using CANScript ${ }^{\mathrm{TM}}$ platform. Cancer Res 75: 1304.

54. Horvat TZ, Adel NG, Dang TO, Momtaz PA, Postow MA, et al. (2015) Immune-related adverse events, need for systemic immunosuppression, and effects on survival and time to treatment failure in patients with melanoma treated with Ipilimumab at Memorial Sloan Kettering Cancer Center. J Clin Oncol 33: 3193-3198. 